\title{
Intussusception non-operative reduction outcome indices (response to "Sonography-guided hydrostatic reduction of ileocolic intussusception in children: analysis of failure and success in consecutive patients presenting timely to the hospital")
}

\author{
Basil Bekdash \\ Received: 26 November 2014 / Accepted: 4 December 2014 / Published online: 23 December 2014 \\ (C) Springer-Verlag Berlin Heidelberg 2014
}

Dear Editors,

I write in response to the article by Menke and Kahl [1] who are the first to publish using our proposed reporting indices since their original publication [2]. We hope that they will prove to be useful to others and ultimately become a standard for reporting outcomes in the management of intussusception or at the very least provoke a discussion as to what the best measures should be.

To clarify, for the benefit of your readers, the 'composite reduction rate' (CoRR) is the best compromise statistic for reporting outcomes. This is because, in principle, it gives a robust and less biased value for the success of non-operative reduction.

We acknowledge that all the defined indices including the CoRR are influenced by at least two variables (e.g. prevalence of disease requiring resection, case selection, technical competence). None of the indices is a pure measure of any one of these parameters without further qualifications/restrictions. The purpose of any outcome measure is to allow fair comparison and flag potential problems and so, the primary reporting index does not necessarily need to indicate the cause, which would be determined by further study.

Communicated by Jaan Toelen

B. Bekdash $(\square)$

Paediatric Surgery Unit, Sheffield Children's Hospital, Sheffield S10

2TH, UK

e-mail: basil.bekdash@gmail.com

Present Address:

B. Bekdash

Nuffield Department of Surgical Sciences, University of Oxford, Level 6 John Radcliffe Hospital, Headley Way, Headington OX3 9DU, UK
I would add that some of the surgical interventions (groups B2, C2, D2) are controversial and may not strictly have been necessary (appendicectomy, caecal fixation; Table 3) with slight influences on the reported values. The categorisation of patients therefore remains a potential source of bias; hence, our suggestion that bowel ischaemia primarily define the need for resection. The authors are to be congratulated for an impressive level of success with the hydrostatic sonographic technique in their retrospective series. As your readers will be aware, there is a lack of strong evidence in this area, partly due to the relative rarity in European and North American settings where much of the literature is generated.

\section{References}

1. Menke J, Kahl F (2014) Sonography-guided hydrostatic reduction of ileocolic intussusception in children: analysis of failure and success in consecutive patients presenting timely to the hospital. Eur J Pediatr

2. Bekdash B, Marven SS, Sprigg A (2013) Reduction of intussusception: defining a better index of successful non-operative treatment. Pediatr Radiol 43:649-656 